\title{
Lupus Panniculitis
}

National Cancer Institute

\section{Source}

National Cancer Institute. Lupus Panniculitis. NCI Thesaurus. Code C82884.

A condition primarily affecting the subcutaneous adipose tissues, showing firm nodules that often resolve with lipoatrophy. There can be overlying changes of discoid lupus erythematosus. A skin biopsy showing lobular panniculitis with typical changes of lupus is needed to rule out other causes of panniculitis, including subcutaneous panniculitis-like Tcell lymphoma. 